\title{
Highly stable two-dimensional metal-carbon monolayer with interpenetrating honeycomb structures
}

\author{
Shulong Li $\mathbb{D}^{1,2,4}$, Kah-Meng Yam $\mathbb{D}^{1,3,4}, \mathrm{Na} \mathrm{Guo}{ }^{1}$, Yong Zhao $\mathbb{D}^{2 凶}$ and Chun Zhang $\mathbb{D}^{1,3 凶}$
}

With the ongoing effort in proposing and realizing functional two-dimensional (2D) materials, we predict by first-principles calculations a family of $2 \mathrm{D}$ metal-carbon $(\mathrm{M}-\mathrm{C})$ crystals consisting of $\mathrm{M}-\mathrm{C}$ trigonal lattice interpenetrated with the metal buckled honeycomb structure. We suggest by simulations that the $2 \mathrm{D} \mathrm{M}-\mathrm{C}$ crystals can be readily fabricated by a self-organizing lattice reconstruction process after placing metal atoms on hollow sites of $\gamma$-graphyne. In total, we found 12 members of the family and they exhibit a variety of electronic and magnetic properties. In this work, we highlight and focus on the Fe member of the family, $2 \mathrm{D}-\mathrm{Fe}_{2} \mathrm{C}_{12}$. Each Fe in $2 \mathrm{D}-\mathrm{Fe}_{2} \mathrm{C}_{12}$ has a magnetic moment of $1 \mu_{\mathrm{B}}$ due to the spin splitting of Fe E1 bands at Fermi surface, resulting in half metallicity and high catalytic activity with unusually high-density single-atom Fe active sites. Ab initio molecular dynamics simulations revealed that the $2 \mathrm{D}-\mathrm{Fe}_{2} \mathrm{C}_{12}$ retains its structural integrity up to $700 \mathrm{~K}$ of simulated short duration annealing. We expect these results to stimulate experimental research for the $2 \mathrm{D} \mathrm{M}-\mathrm{C}$ crystals we proposed.

npj 2D Materials and Applications (2021)5:52; https://doi.org/10.1038/s41699-021-00235-y

\section{INTRODUCTION}

Since the rise of graphene ${ }^{1,2}$, two-dimensional (2D) materials have attracted enormous interest in recent years owing to their unique properties and great potential in various applications. Discovering 2D functional materials beyond graphene ${ }^{3,4}$ that have exotic physical and chemical properties lies at the heart of the current research effort along this direction. Examples of these 2D materials include $\mathrm{h}-\mathrm{BN}^{5,6}, \mathrm{~g}-\mathrm{C}_{3} \mathrm{~N}_{4}{ }^{7,8}$, and $2 \mathrm{D}$ transition-metal dichalcogenides $^{9,10}$ that are semiconductors with sizeable bandgaps, and 2D$\mathrm{Cu}_{2} \mathrm{Si}$ monolayer ${ }^{11,12}$ that is a metal with high conductivity. With the ongoing effort of looking for new functional 2D materials, 2D carbon-based networks, such as porphyrin and phthalocyanine systems ${ }^{13,14}$, have been widely considered as an ideal template for realizing emerging $2 \mathrm{D}$ properties because of the high stability and flexibility of the network. The reason behind the stability of such systems is owing to the presence of $\mathrm{N}$ atoms that not only form strong bonds with $\mathrm{C}$ atom but also interact favorably with metal atoms in porphyrin and phthalocyanine. On the other hand, interactions between metal and $C$ atoms tend to be much weaker. The evidence for this is that doping of carbon-based materials like graphene and carbon nanotubes with metal atoms result in poor stability of the dopants, leading to sintering and formation of metal nanoclusters, as indicated from various theoretical studies and experimental observations ${ }^{15-18}$. As a result, vacancies in carbon substrate like graphene and/or other impurity atoms are necessary for experiments to stabilize the 2D carbon-metal systems ${ }^{19-21}$. It is then of special significance that we, here in this paper, propose a carbon framework involving metal-carbon $(\mathrm{M}-\mathrm{C})$ bonds, which can give rise to a family of highly stable 2D crystals. The $2 \mathrm{D} \mathrm{M}-\mathrm{C}$ crystal has a structure that has two metals and $12 \mathrm{C}$ in one unit cell (with a chemical formula $\mathrm{M}_{2} \mathrm{C}_{12}$ ) and consists of both $M-C_{5}$ and $C_{6}$ hexagonal rings. Metal atoms themselves form a periodic honeycomb pattern embedded in a graphene honeycomb lattice, which we call in this paper the Honeycomb-in-Honeycomb $(\mathrm{HIH})$ structure.
With different choices of metal atoms, 2D-M $\mathrm{C}_{12}$ exhibits a variety of electronic and magnetic properties. We show that the $2 \mathrm{D}-\mathrm{M}_{2} \mathrm{C}_{12}$ can be metal or semiconductor, and magnetic or nonmagnetic. In particular, in this paper, we highlight and focus on the Fe member of the family, $2 \mathrm{D}-\mathrm{Fe}_{2} \mathrm{C}_{12}$, which is a half-metal with $1 \mu_{\mathrm{B}}$ of magnetic moment on each Fe. Great research efforts have been made in recent years in inducing magnetism in $2 \mathrm{D}$ materials ${ }^{22-25}$. Here, we show that the $2 D M-C$ crystals, for example $2 \mathrm{D}-\mathrm{Fe}_{2} \mathrm{C}_{12}$, could be a avenue for making $2 \mathrm{D}$ magnetic materials that have potential for nanoscale spintronic applications such as spin filter and transistors. We will further show that the extremely high density of exposed $\mathrm{Fe}$ atoms in $2 \mathrm{D}-\mathrm{Fe}_{2} \mathrm{C}_{12}$ can function as catalytic active centers. $2 \mathrm{D}$ carbon-based materials have attracted considerable attention as a host material for impregnating catalytically active metal atoms as single-atom catalysts $(\mathrm{SACS})^{19-21,26}$. For $2 \mathrm{D}-\mathrm{Fe}_{2} \mathrm{C}_{12}$, unlike most of previously proposed SACs, the catalytic activity is intrinsic and not by modifying local properties of the system. Also, sintering of the catalysts resulting from the agglomeration of the dopants subjected to the harsh operating conditions can be avoided, therefore, the stability of $2 \mathrm{D}-\mathrm{Fe}_{2} \mathrm{C}_{12}$ manifests as an important advantage over many other SACs.

\section{RESULTS AND DISCUSSIONS Design of $2 \mathrm{D}-\mathbf{M}_{\mathbf{2}} \mathbf{C}_{\mathbf{1 2}}$}

The conception of such material design is built upon the basis that graphyne (GY), a family of allotropes of 2D carbon consisting of a mixture of $s p$ - and $s p^{2}$-hybridized $C$ atoms, offers various degrees of porosity ${ }^{27,28} \cdot \gamma^{-G Y}$ has been successfully synthesized via various bottom-up approaches ${ }^{27,29}$. Previous theoretical studies have shown that it is a good host material for metal atoms, with the triangular hollow sites shown in Fig. 1 as good-trapping sites ${ }^{30}$. The motivation of this work is that if the $M-C$ interaction is sufficiently strong, a significant lattice reconstruction could occur

\footnotetext{
${ }^{1}$ Department of Physics and Centre for 2D Advanced Materials, National University of Singapore, Singapore, Singapore. ${ }^{2}$ Key Laboratory of Magnetic Levitation Technologies and Maglev Trains (Ministry of Education), Superconductivity and New Energy R\&D Center, Southwest Jiaotong University, Chengdu, China. ${ }^{3}$ Department of Chemistry, National University of Singapore, Singapore, Singapore. ${ }^{4}$ These authors contributed equally: Shulong Li, Kah-Meng Yam. ${ }^{\circledR}$ email: zhaoyong@fjnu.edu.cn; phyzc@nus.edu.sg
} 


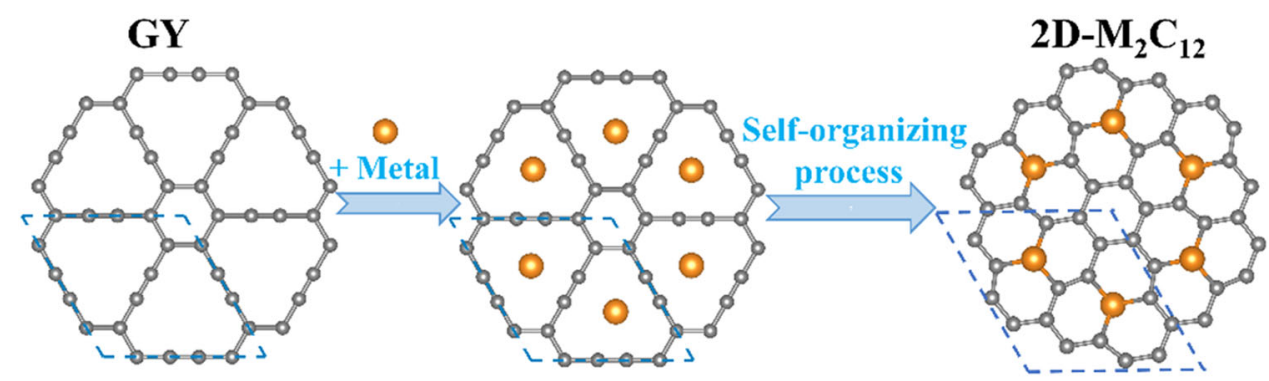

Fig. 1 The possible mechanism for the synthesis of $\mathbf{2 D}-\mathbf{M}_{\mathbf{2}} \mathbf{C}_{\mathbf{1 2}}$ from $\mathbf{G Y}$. The triangular hollow sites in GY are good-trapping sites for the metal atoms. Upon adsorption, the strong metal-carbon interactions induce lattice reconstruction in a self-organizing process forming $2 \mathrm{D}-\mathrm{M}_{2} \mathrm{C}_{12}$. Unit cells are enclosed in cyan dashed lines.
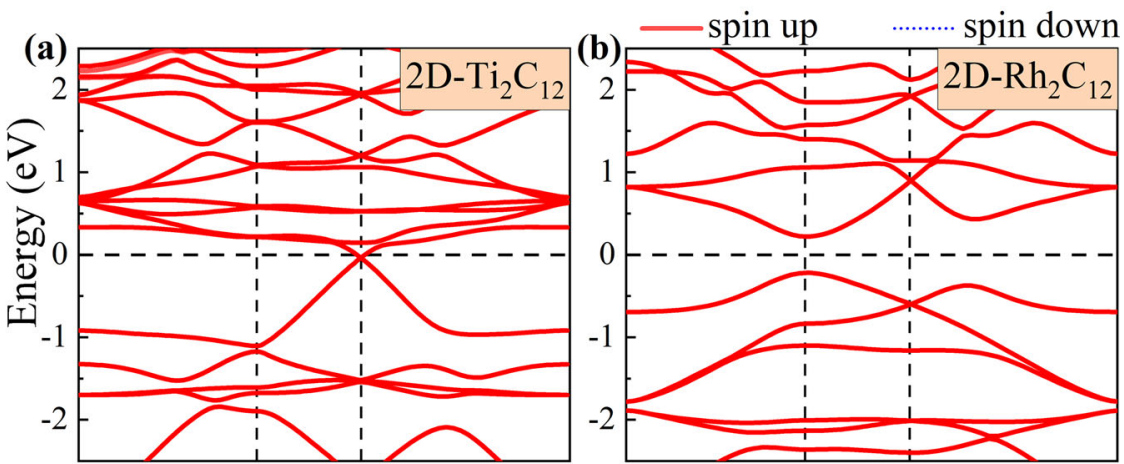

(c)

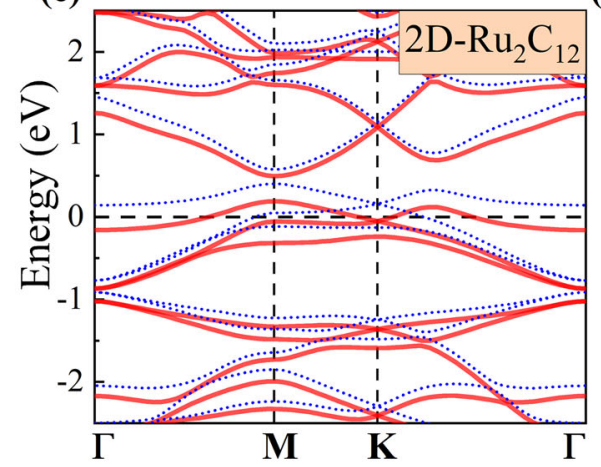

(d)

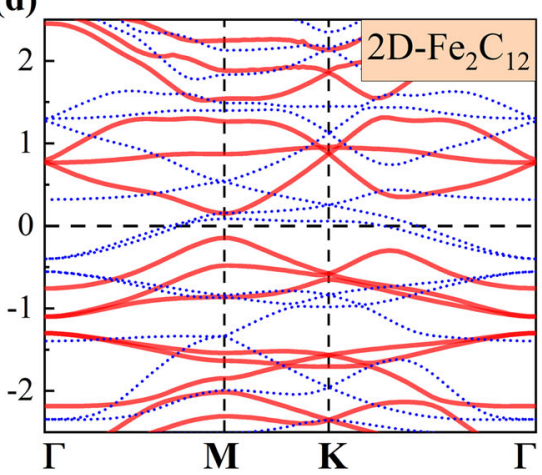

Fig. 2 Electronic band structures of $2 \mathrm{D}-\mathbf{M}_{\mathbf{2}} \mathrm{C}_{\mathbf{1 2}}$. The band structures of a $2 \mathrm{D}-\mathrm{Ti}_{2} \mathrm{C}_{12}$, a nonmagnetic metal; $\mathbf{b} 2 \mathrm{D}-\mathrm{Rh} \mathrm{C}_{12}$, a nonmagnetic semiconductor with a bandgap of $0.45 \mathrm{eV}$ at $\mathrm{M}$ point; $\mathbf{c}$ the magnetic and metallic $2 \mathrm{D}-\mathrm{Ru}_{2} \mathrm{C}_{12}$; and lastly, $\mathbf{d}$ the half-metallic $2 \mathrm{D}-\mathrm{Fe} \mathrm{C}_{2} \mathrm{C}_{12}$ with a semiconducting spin-up channel of quasi bandgap $0.30 \mathrm{eV}$ at $\mathrm{M}$ point. $\mathbf{c}$, $\mathbf{d}$ spin-up (-down) bands are in red (blue).

after metal atoms being trapped, and then each highly reactive $\mathrm{C}$ $C$ triple bond can break one of its $\pi$ bonds to facilitate $\mathrm{M}-\mathrm{C}$ bond formation, resulting in a tri-coordinated metal atom and the formation of $2 \mathrm{D}-\mathrm{M}_{2} \mathrm{C}_{12}$ as shown in Fig. 1. To verify this, we performed density functional theory (DFT) based first-principles calculations. We first obtained the atomic and band structures of GY, as shown in Supplementary Figure 1 in supporting information, which agree very well with previous reports ${ }^{28}$. Then, each metal atom was placed at one of the triangular hollow sites and the ground-state structure of the monolayer was obtained using full relaxation without any symmetry constraints. We tested various metal species, for 12 of them (e.g., Ti, Cr, Mn, Fe, Cu, Mo, $\mathrm{Ru}, \mathrm{Rh}, \mathrm{W}, \mathrm{Re}, \mathrm{Os}, \mathrm{Ir}$ ), the carbon skeleton prefer undergoing a lattice reconstruction and the graphynic lattice is no longer visible, leading to a self-organized periodic honeycomb pattern of metal atoms in $2 \mathrm{D}-\mathrm{M}_{2} \mathrm{C}_{12}$ (Fig. 1). Note that in the lattice reconstruction process, there is no substitution of $\mathrm{C}$ atoms by metal atoms as evident in Supplementary Video 1.

\section{Electronic structures of $2 \mathrm{D}-\mathrm{M}_{\mathbf{2}} \mathrm{C}_{\mathbf{1 2}}, \mathbf{M}=\mathrm{Ti}, \mathbf{R h}, \mathbf{R u}, \mathrm{Fe}$}

The $2 \mathrm{D}-\mathrm{M}_{2} \mathrm{C}_{12}$ monolayer shows a variety of electronic structures with different choices of metal atoms. Here, we show $\mathrm{Ti}, \mathrm{Rh}, \mathrm{Ru}$, and Fe members as four typical cases. The resulting optimized atomic structures of all four systems resembles the graphenic network with a trigonal lattice of $P$-3 space group. Nevertheless, these materials exhibit very different electronic properties evident from the band structure of each system shown in Fig. 2, in which $2 \mathrm{D}-\mathrm{Ti}_{2} \mathrm{C}_{12}$ is a nonmagnetic metal, 2D$\mathrm{Rh}_{2} \mathrm{C}_{12}$ is a nonmagnetic semiconductor with a direct bandgap of $0.45 \mathrm{eV}$ at $\mathrm{M}$ point, $2 \mathrm{D}-\mathrm{Ru}_{2} \mathrm{C}_{12}$ is a magnetic metal with a 0.31 $\mu_{\mathrm{B}}$ of magnetic moment on each Ru atom, and $2 \mathrm{D}-\mathrm{Fe}_{2} \mathrm{C}_{12}$ is half metal with a semiconducting spin-up channel of quasi bandgap $0.30 \mathrm{eV}$ at $\mathrm{M}$ point, and $\sim 1 \mu_{\mathrm{B}}$ of magnetic moment resides on each Fe atom. The highly tunable electronic structure of 2D$\mathrm{M}_{2} \mathrm{C}_{12}$ makes the material useful for various kinds of applications. In this work, we are particularly interested in half-metallic $2 \mathrm{D}-\mathrm{Fe}_{2} \mathrm{C}_{12}$. 
Atomic structure of $2 \mathrm{D}-\mathrm{Fe}_{\mathbf{2}} \mathrm{C}_{\mathbf{1 2}}$

The atomic structure of the relaxed $2 \mathrm{D}-\mathrm{Fe}_{2} \mathrm{C}_{12}$ is shown in Fig. $3 \mathrm{a}$, which is also very similar for the Ti-, Rh, and Ru-systems, forming a trigonal lattice with $P-3$ space group in the 2D plane. The optimized lattice constants are obtained by fitting using the third order Birch-Murnaghan equation of state ${ }^{31}$ shown in Supplementary Figure $2 a$, giving $a=b=6.75 \AA$. There are two different types of $C$ atoms, which we will denote as $C^{1}$ (bonded with Fe) and $C^{2}$ (the rest), respectively, although both are $s p^{2}$ hybridized. The calculated bond lengths for $C^{1}-C^{1}, C^{2}-C^{2}$, and $C^{1}-C^{2}$ are $1.39 \AA$, $1.41 \AA$, and $1.43 \AA$, respectively. Each $\mathrm{Fe}$ is threefold-coordinated with $C^{1}$ atoms and the calculated $C^{1}-$ Fe bond length is $1.80 \AA$. The material shows an interesting $\mathrm{HIH}$ structure as aforementioned.

It is noteworthy that the configuration with two Fe atoms that protrude in the same direction (atomic structure shown in Supplementary Figure 2b) were also tested but is $10 \mathrm{meV}$ higher in energy than the ground-state structure. Nevertheless, the electron localization function plot in Supplementary Figure 3 shows significant hybridization between $\mathrm{C}^{1}$ and Fe atoms and these favorable interactions stabilizes the Fe atoms, which we will show shortly, and results in the magnetic moment largely localized on the Fe atoms, as supported from the spin differential density plotted in Fig. 3b.

\section{Stability of $2 \mathrm{D}-\mathrm{Fe}_{\mathbf{2}} \mathrm{C}_{12}$}

The stability of $2 \mathrm{D}-\mathrm{Fe}_{2} \mathrm{C}_{12}$ was preliminarily tested by calculating the cohesive energy $\left(E_{\mathrm{coh}}\right)$ and the formation energy $\left(E_{\mathrm{form}}\right)$. The cohesive energy was estimated to be $6.86 \mathrm{eV}$ atom $^{-1}$. For comparison, the $E_{\text {coh }}$ of other $2 \mathrm{D}$ materials were also calculated and tabulated in Table 1. The high value of $E_{\text {coh }}$ shows that the stability of $2 \mathrm{D}-\mathrm{Fe}_{2} \mathrm{C}_{12}$ is at least comparable to materials like silicene, 2D-Cu $2 \mathrm{Si}$ and even monolayer $\mathrm{MoS}_{2}$. Furthermore, the formation energy of $2 \mathrm{D}-\mathrm{Fe}_{2} \mathrm{C}_{12}$ from $\mathrm{GY}$ and Fe atoms is calculated to be $-0.56 \mathrm{eV}$ atom $^{-1}$. Such exothermicity should be indicative of the facile formation of $2 \mathrm{D}-\mathrm{Fe}_{2} \mathrm{C}_{12}$. The structural stability of $2 \mathrm{D}$ $\mathrm{Fe}_{2} \mathrm{C}_{12}$ was ascertained by the calculated phonon dispersion with all the vibrational modes being real in the entire Brillouin zone (BZ) as plotted in Fig. 3c. A noteworthy feature is that the phonon branches occurring above $v=63.0 \mathrm{THz}$ in $\mathrm{GY}^{32}$ are absent because the $\mathrm{C} \equiv \mathrm{C}$ were broken so that the $\mathrm{C}^{1}$ atoms can hybridize with $\mathrm{Fe}$ states.

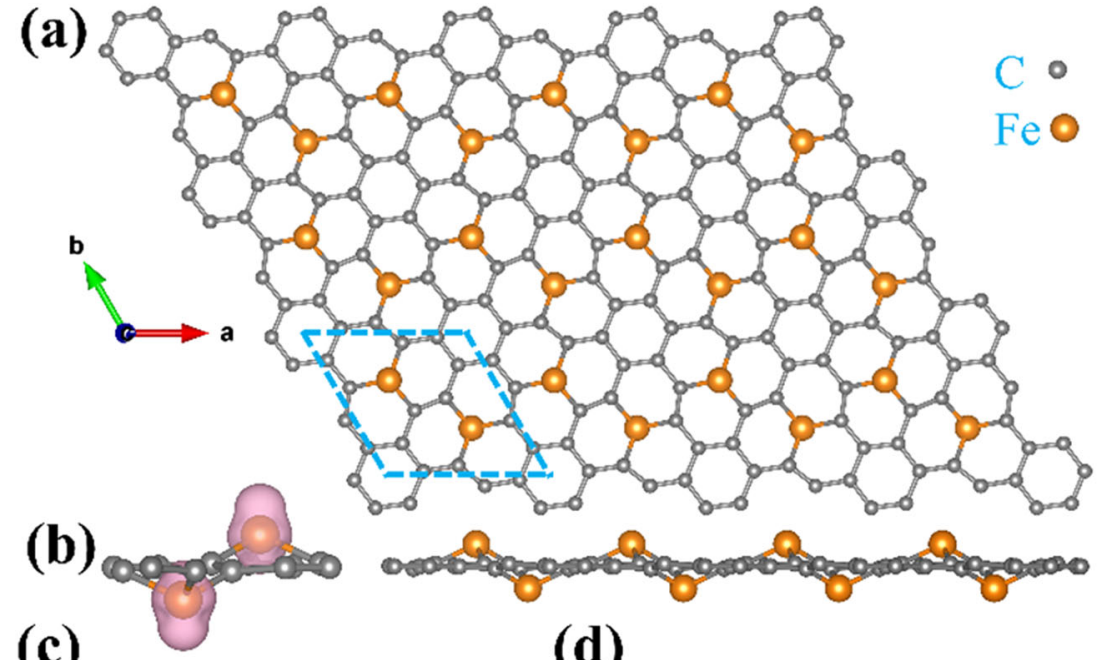

(c)

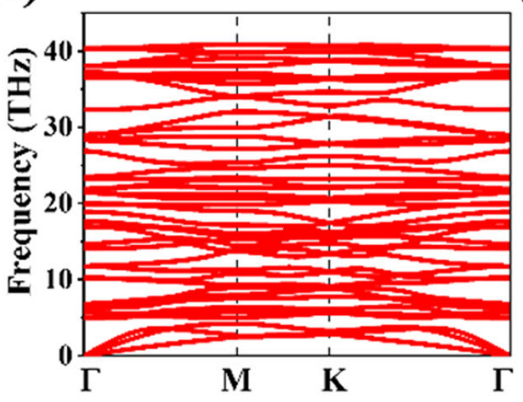

(d)

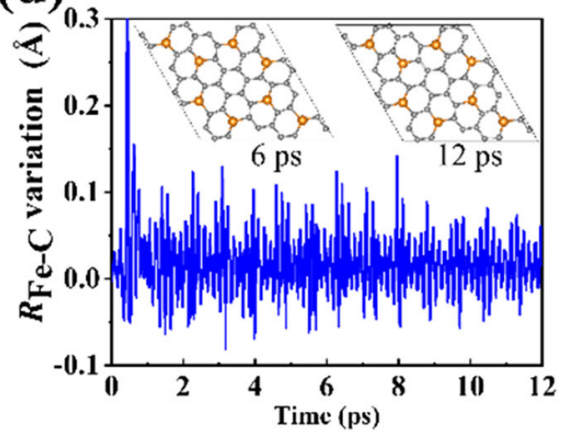

Fig. 3 Atomic structure and stability of $2 \mathrm{D}-\mathrm{Fe}_{2} \mathbf{C}_{\mathbf{1 2}}$. a Top- and side-view of the optimized $2 \mathrm{D}-\mathrm{Fe}_{2} \mathrm{C}_{12}$ monolayer with the primitive cell enclosed in cyan dashed lines. $\mathbf{b}$ The spin differential density plot of $2 \mathrm{D}-\mathrm{Fe}_{2} \mathrm{C}_{12}$, showing that the magnetic moment is largely localized on the Fe atoms. $c$ The phonon dispersion spectrum of $2 \mathrm{D}-\mathrm{Fe}_{2} \mathrm{C}_{12}$. $\mathbf{d}$ Results of the AIMD calculation showing the Fe-C bond length variation at $300 \mathrm{~K}$. Insets are snapshots of atomic structures at 6 and $12 \mathrm{ps}$.

Table 1. Lattice constant $(a=b)$ and cohesive energy $\left(E_{\text {coh }}\right)$ of $2 \mathrm{D}-\mathrm{Fe}_{2} \mathrm{C}_{12}$ and other $2 \mathrm{D}$ materials.

\begin{tabular}{lllllllll}
\hline & $2 \mathrm{D}-\mathrm{Fe}_{2} \mathrm{C}_{12}$ & $\mathrm{GY}$ & Graphene & Silicene & 2D-Cu $\mathrm{Ci}_{2}$ & $\mathrm{~g}-\mathrm{C}_{3} \mathrm{~N}_{4}$ & $\mathrm{MoS}_{2}$ & $\mathrm{~h}-\mathrm{BN}$ \\
\hline$a=b(\AA)$ & 6.75 & 6.89 & 2.46 & 3.87 & 4.12 & 7.14 & 3.18 \\
$E_{\text {coh }}\left(\mathrm{eV}\right.$ atom $\left.^{-1}\right)$ & 6.86 & 7.32 & 7.96 & 3.96 & 3.65 & 6.35 & 5.14 & 7.09 \\
\hline
\end{tabular}


The temperature-dependent stability of the material was assessed by the ab initio molecular dynamics (AIMD) simulations. The corresponding trajectories and the snapshots of the geometries at different junctures are shown in Supplementary Figure 4, with the variation of the $\mathrm{Fe}-\mathrm{C}^{1}$ bonds and the snapshots at 6 and $12 \mathrm{ps}$ at $300 \mathrm{~K}$ shown in Fig. 3d. It is evident that the material is very stable, with the Fe atoms showing no signs of undergoing any form of significant migration. From a series of AIMD simulations (Supplementary Figure 4), the atomic network is well-maintained at the temperatures of up till $700 \mathrm{~K}$, at which 2D$\mathrm{Fe}_{2} \mathrm{C}_{12}$ could survive a $12 \mathrm{ps}$ annealing up to $700 \mathrm{~K}$. At $800 \mathrm{~K}$, the out-of-plane vibrations of $\mathrm{Fe}$ atoms are violent enough to break the $\mathrm{Fe}-\mathrm{C}^{1}$ bonds, resulting in the destruction of the network consisting of $\mathrm{FeC}_{5}$ rings and the partial restoration of the GY carbon skeleton, indicating the decomposition of the material. It is worth mentioning here that the error of the AIMD calculations increases with temperature, but based on our results, it is safe to conclude that the material is highly stable under room temperature.

\section{Magnetic coupling in $2 \mathrm{D}-\mathrm{Fe}_{\mathbf{2}} \mathrm{C}_{\mathbf{1 2}}$}

We next study the magnetic coupling between the $\mathrm{Fe}$ atoms in calculating the total energies of the ferromagnetic (FM) and antiferromagnetic (AFM) configuration in one unit cell, and also two other AFM configurations in a $1 \times 2$ supercell (AFM2 and AFM3 in Supplementary Figure 5). It was found that the FM configuration is $88,35,13$, and $103 \mathrm{meV}$ lower in energy than the AFM, AFM2, AFM3 and the nonmagnetic configuration per unit cell, respectively, indicating that the Fe atoms prefer to couple ferromagnetically. The calculated magnetic moment for the FM configuration is $\sim 2 \mu_{\mathrm{B}}$ per unit cell, with $1 \mu_{\mathrm{B}}$ residing on each Fe atom, as shown from the isosurface plot of the spin density.

\section{Origin of magnetism in $2 \mathrm{D}-\mathrm{Fe}_{\mathbf{2}} \mathrm{C}_{\mathbf{1 2}}$}

The magnetism of $2 \mathrm{D}-\mathrm{Fe}_{2} \mathrm{C}_{12}$ can be elucidated from its band structure. The energy bands responsible for the magnetism is shown in Fig. 4a. Those bands show an intriguing half-metallic behavior as aforementioned. Half metals have great potential in spintronics applications such as spin filtering and transistors that have been extensively reported in literature ${ }^{33-35}$. The half-metallic behavior of $2 \mathrm{D}-\mathrm{Fe}_{2} \mathrm{C}_{12}$ provides more opportunities for future design of $2 \mathrm{D}$ spintronic devices. In the absence of relatively flat impurity bands in the $B Z$, the bands in the vicinity of Fermi energy $\left(E_{\mathrm{F}}\right)$ are largely dominated by those originated from the hybridization between the Fe $d$ orbitals and the $\pi$ bands of the carbon skeletal network. Despite this strong hybridization, the calculated magnetic moment would originate from the occupation of the largely non-bonding energy bands ${ }^{30}$, which we have identified and labeled by their symmetry as A, E1, and E2 in Fig. 4a. The symmetry of bands is identified by plotting the banddecomposed charge densities (Fig. 4b). There are five such nonbonding bands (two E2, two E1, and one A) in each spin channel near $E_{\mathrm{F}}$ in the range of -1.75 to $-0.20 \mathrm{eV}$ for the spin-up channel and -1.00 to $0.50 \mathrm{eV}$ for spin-down. The bands $A$ is the highest in energy, followed by E1 and E2. Such order in energy can be understood by $d$ orbital splitting and hybridization of threefold coordinated $\mathrm{Fe}$ atoms in a $\mathrm{C}_{3 \mathrm{v}}$ ligand field (see Supplementary Notes in supporting information for more detailed analysis).

To work out the occupation of those bands, we noted that for the two Fe with 16 valence electrons in consideration (with each Fe contributing six $3 d$ and two $4 s$ electrons), six of these electrons are required to form $\sigma$ bonds with the $C^{1}$ atoms and two more to maintain the $\pi$ band. The remaining eight electrons would fill up the largely non-bonding bands mentioned A, E1, and E2. All five of them in the spin-up channel would be filled, with the quasivalence band (VB) mainly composed of the Fe $d_{z^{2}}$, as corroborated from the projected density of states (PDOS) and the banddecomposed density. The remaining three electrons would fill up the bands E1 and E2 in the spin-down channel, with bands E1 crossing $E_{\mathrm{F}}$ and approximately half filled. As such, this will leave two unpaired electrons giving rise to the magnetic moment of 2 $\mu_{\mathrm{B}}$ per unit cell. The results can be summarized in a simple schematic of non-bonding orbitals (shown in Fig. 4c) that represents the largely non-bonding bands, with the order of the orbitals obtained from the energies of the non-bonding bands at $\Gamma$ point. Note that the spin-down E1 bands cross $E_{\mathrm{F}}$ so we put the two spin-down E1 orbitals right at $E_{\mathrm{F}}$ and make them half filled, which finally lead to $2 \mu_{\mathrm{B}}$ of magnetic moment in one unit cell. This non-bonding orbital model is also capable of deducing the

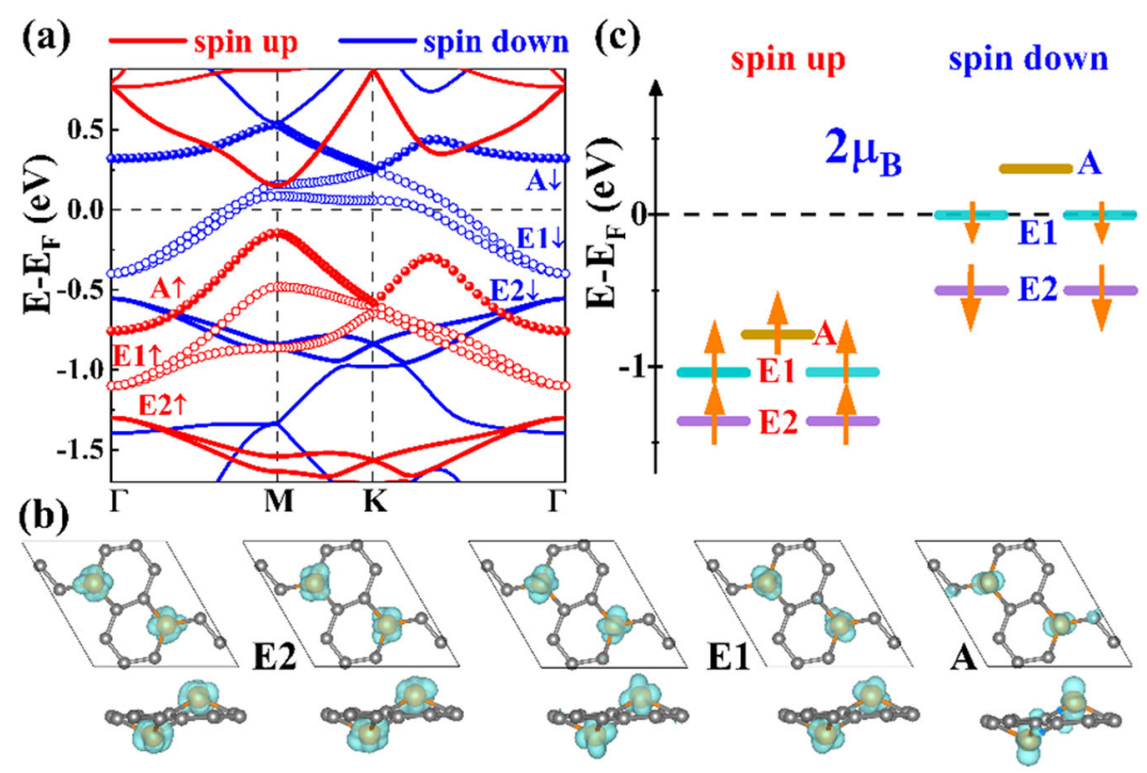

Fig. 4 Electronic structure analyses for $2 \mathrm{D}-\mathrm{Fe}_{2} \mathrm{C}_{\mathbf{1 2}}$. a The spin-polarized band structure of $2 \mathrm{D}-\mathrm{Fe}_{2} \mathrm{C}_{12}$. Fe bands near Fermi are labeled by their symmetry $A, E 1$, and E2. $\mathbf{b}$ Band-decomposed charge density of the largely non-bonding bands at $\Gamma$ point, which are responsible for the origin of the magnetic moment. c A simple schematic of non-bonding orbitals that represents the largely non-bonding bands A, E1, and E2. Note that the spin-down E1 orbitals are half filled. 

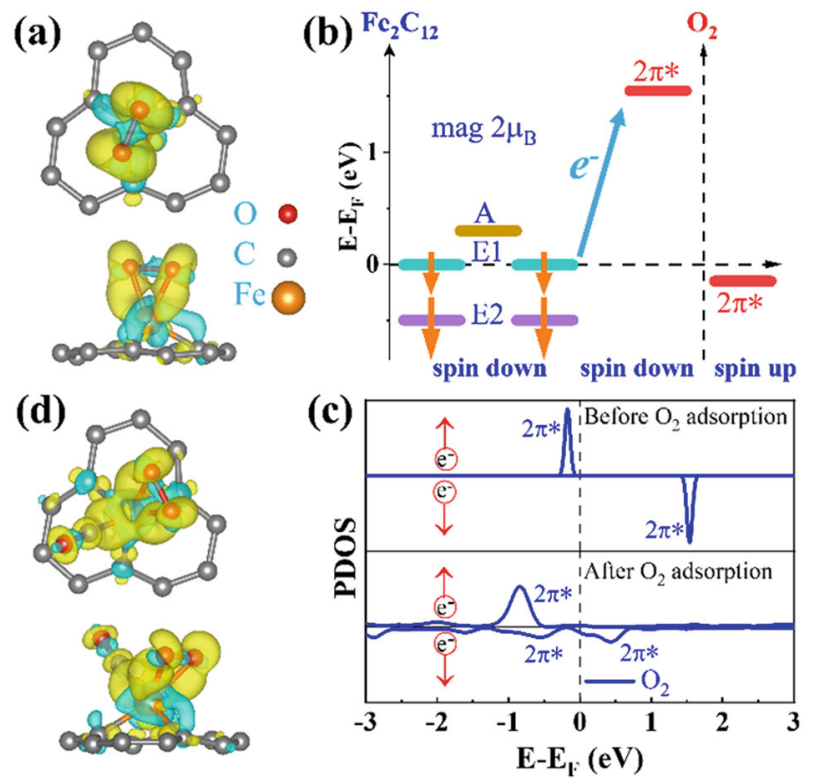

Fig. 5 Intrinsic reactivity of $2 \mathrm{D}-\mathrm{Fe}_{2} \mathrm{C}_{12}$. a Charge differential density plots upon $\mathrm{O}_{2}$ adsorption on $2 \mathrm{D}-\mathrm{Fe}_{2} \mathrm{C}_{12}$, respectively, with the yellow (cyan) isosurface representing charge accumulation (depletion). b Schematic showing the magnetism-induced catalytic activity of $2 \mathrm{D}-\mathrm{Fe}_{2} \mathrm{C}_{12}$, transferring the electron in the partially filled Fe $\mathrm{E} 1$ band in the spin-down channel to $\mathrm{O}_{2}$. c The PDOS of $\mathrm{O}_{2}$ before and after adsorption. $\mathbf{d}$ Charge redistribution induced by $\mathrm{CO}$ and $\mathrm{O}_{2}$ co-adsorption on $2 \mathrm{D}-\mathrm{Fe}_{2} \mathrm{C}_{12}$.

magnetic moments calculated in other members of the family. The band structure and the spin density suggest that the magnetic Fe centers can be excellent electron donors. The halffilled conduction bands E1 of the spin-down channel crosses the $E_{\mathrm{F}}$, which are ready to transfer electron to an acceptor, suggesting that besides spintronics applications, the material may also be catalytically active.

\section{Catalytic activity of $2 \mathrm{D}-\mathrm{Fe}_{2} \mathrm{C}_{12}$}

To study the catalytic activity of $2 \mathrm{D}-\mathrm{Fe}_{2} \mathrm{C}_{12}$, the $\mathrm{CO}$ oxidation reaction (COR) was chosen as a probe reaction. The adsorption of $\mathrm{CO}, \mathrm{O}_{2}$, and $\mathrm{CO}_{2}$ is crucial for understanding the catalytic activity. It was found that $\mathrm{O}_{2}$ and $\mathrm{CO}$ molecules do not adsorb on the $\mathrm{C}$ atoms but can chemisorb strongly on Fe with adsorption energies being -1.32 and $-0.95 \mathrm{eV}$, respectively, with the most stable adsorption configurations shown in Fig. $5 \mathrm{a}\left(\mathrm{O}_{2}\right)$ and S8 (CO). $\mathrm{O}_{2}$ takes a side-on adsorption on $\mathrm{Fe}$, with a sizeable charge transfer of $\sim 0.78 \mathrm{le} \mid$ from Fe to the $2 \pi^{*}$ antibonding orbital of $\mathrm{O}_{2}$ as indicated by the charge redistribution iso-surfaces (Fig. 5a). In Fig. 5b, we show the energy levels (near $E_{\mathrm{F}}$ ) of the $\mathrm{Fe}$ and $\mathrm{O}_{2}$ before adsorption. As suggested by the figure, the $<1|\mathrm{e}|$ charge transfer shall happen from the partially filled Fe E1 spin-down orbital to the empty spin-down $2 \pi^{*}$ orbital of $\mathrm{O}_{2}$. Such charge transfer is verified by the PDOS of $\mathrm{O}_{2}$ before and after adsorption plotted in Fig. 5c. Before adsorption, the spin-down $2 \pi^{*}$ orbital of isolated $\mathrm{O}_{2}$ is unoccupied. After adsorption, the spin-down $2 \pi^{*}$ orbital split to two peaks, with one of them is pulled below Fermi level, indicating the partial charge transfer onto the $2 \pi^{*}$ orbital. The charge transfer decreases the magnetic moment of $\mathrm{O}_{2}$ from 2.0 to $0.55 \mu_{\mathrm{B}}$ and leads the $\mathrm{O}-\mathrm{O}$ bond significantly elongated from 1.23 to $1.40 \AA$. Compared with $\mathrm{O}_{2}$, CO-adsorption is weaker with a smaller $0.29|\mathrm{e}|$ transferred from the Fe to $\mathrm{CO}$. At last, $\mathrm{CO}_{2}$ does not adsorb on any $C$ sites and only recorded a very weak adsorption energy of $-0.03 \mathrm{eV}$ on Fe (see adsorption structure in Supplementary Figure $8 \mathrm{~b}$ ), implying that the $\mathrm{CO}_{2}$ molecules can spontaneously desorb from $\mathrm{Fe}$ under room temperature.

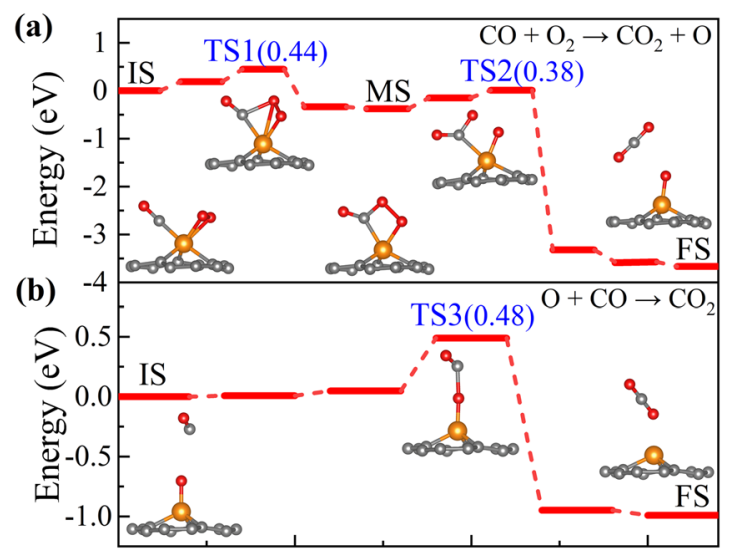

Reaction coordinate

Fig. 6 Energy profiles of $\mathrm{CO}$ oxidation reaction. a The first step via $\mathrm{LH}$ mechanism, $\mathrm{CO}+\mathrm{O}_{2} \rightarrow \mathrm{CO}_{2}+\mathrm{O}$. b The second step, $\mathrm{O}+\mathrm{CO} \rightarrow$ $\mathrm{CO}_{2}$. Atomic structures of the initial (IS), transition (TS), and final (FS) states are shown. Energy barriers (in $\mathrm{eV}$ ) needed to reach TS are given in brackets. Color scheme used is the same as before.

We also considered the adsorption of $\mathrm{CO}$ with one $\mathrm{O}_{2}$ molecule pre-adsorbed on Fe. We found that in this case, the adsorption energy of $\mathrm{CO}$ is $\sim-0.32 \mathrm{eV}$. The co-adsorption configuration with the charge redistribution induced by both $\mathrm{CO}$ and $\mathrm{O}_{2}$ is shown in Fig. $5 \mathrm{~d}$. The analysis of $\mathrm{CO}, \mathrm{O}_{2}$, and $\mathrm{CO}_{2}$ adsorptions suggests that the catalyzed COR can happen in both the Eley-Rideal (ER) and the Langmuir-Hinshelwood (LH) mechanisms. Indeed, our calculations show that both mechanisms are possible, whereas the $\mathrm{LH}$ mechanism is favored with a low reaction barrier $<0.5 \mathrm{eV}$. For the LH mechanism, as shown in Fig. 6a, in the calculated first step of the reaction ( $\left.\mathrm{CO}+\mathrm{O}_{2} \rightarrow \mathrm{CO}_{2}+\mathrm{O}\right)$, the reaction proceeds initially with the rotation of the adsorbed $\mathrm{O}_{2}$ molecule to form the peroxotype (OCOO) metastable transition state (TS), with a low reaction barrier $\left(E_{\mathrm{b}}\right)$ of $0.44 \mathrm{eV}$. Then the $\mathrm{O}-\mathrm{O}$ bond increases, requiring only $0.38 \mathrm{eV}$ to form the second TS. Subsequently, the Fe-C bond breaks, releasing a $\mathrm{CO}_{2}$ molecule. This process is highly exothermic with a $\Delta_{\mathrm{r}} E=-3.29 \mathrm{eV}$. For the second step $(\mathrm{O}+$ $\mathrm{CO} \rightarrow \mathrm{CO}_{2}$, shown in Fig. $6 \mathrm{~b}$ ), another $\mathrm{CO}$ comes, reacts with the left $\mathrm{O}$ atom and forms $\mathrm{CO}_{2}$. This step has a reaction barrier of $0.48 \mathrm{eV}$. For the ER mechanism, the calculated energy profile is shown in Supplementary Figure 9, we noted that the gaseous CO molecule attacked the pre-adsorbed and activated $\mathrm{O}_{2}$, reached the TS with $E_{\mathrm{b}}=0.79 \mathrm{eV}$. Then, the second $\mathrm{CO}$ molecule attacks the left $\mathrm{O}$ to form the second $\mathrm{CO}_{2}$ with $E_{\mathrm{b}}=0.48 \mathrm{eV}$. Therefore, 2D$\mathrm{Fe}_{2} \mathrm{C}_{12}$ is an excellent catalyst for COR which will predominantly happen via the $\mathrm{LH}$ path with a low reaction barrier of $0.48 \mathrm{eV}$, indicating that the reaction can happen under room temperature.

Via first-principles calculations, we proposed a family of 2D M-C monolayers with an intriguing $\mathrm{HIH}$ structure in which metal atoms form a periodic honeycomb pattern embedded in graphene honeycomb lattice. The 2D M-C monolayer has a chemical formula $\mathrm{M}_{2} \mathrm{C}_{12}$ and can be obtained by a self-organizing lattice reconstruction process of GY with metal atoms in its hollow triangular sites. In total, we have found 12 members of the family. With different metal, $2 \mathrm{D}-\mathrm{M}_{2} \mathrm{C}_{12}$ show different electronic and magnetic properties, which makes the material useful for various applications. We focused our analysis on the Fe member of the family, $2 \mathrm{D}-\mathrm{Fe}_{2} \mathrm{C}_{12}$, which is a half metal. The structural stability of $2 \mathrm{D}-\mathrm{Fe}_{2} \mathrm{C}_{12}$ were evaluated and we found that it can survive up till $700 \mathrm{~K}$. Detailed analysis reveals that the half-metallic behavior originates from the spin-split of Fe E1 orbital at $E_{\mathrm{F}}$. The half metallicity clearly suggests that the $2 \mathrm{D}-\mathrm{Fe}_{2} \mathrm{C}_{12}$ can be useful for spintronic applications such as spin filtering and spin transistors. We also show that the exposed magnetic Fe sites in $2 \mathrm{D}-\mathrm{Fe}_{2} \mathrm{C}_{12}$ are highly catalytically active towards $\mathrm{CO}$ oxidation. The ultra-high 
density of Fe atoms implies high performance of $2 \mathrm{D}-\mathrm{Fe}_{2} \mathrm{C}_{12}$ as a single-atom site catalyst. We foresee that such metal embedded carbon-based material can also be realized soon with the addition of the appropriate reagents in the reaction mixture and its application potential are awaiting to be verified by experiments.

\section{METHODS}

\section{Structure optimization and electronic structure analysis}

All first-principles calculations were carried out under the spin-polarized DFT formalism. Structure optimization, electronic structure, and total energy calculations were done using the Vienna $A b$ initio Simulation Package (VASP) ${ }^{36,37}$ employing the projector augmented wave pseudopotentials $^{38}$ and a plane wave basis set with a kinetic cutoff energy of $500 \mathrm{eV}$ were used. In addition, the Perdew-Burke-Ernzerhof format ${ }^{39}$ of the generalized gradient approximation was adopted for the exchange-correlation functional. All the structures were relaxed without any symmetry constraints and until the total energy and the residual Hellmann-Feynman force acting on each atom is $<10^{-5} \mathrm{eV}$ and $0.01 \mathrm{eV}$ $\AA^{-1}$, respectively. To eliminate unphysical interaction between the replicas of periodic images, a vacuum space of at least $20 \AA$ in the direction perpendicular to the $2 \mathrm{D}-\mathrm{Fe}_{2} \mathrm{C}_{12}$ atomic plane, i.e., the $z$-direction. The $\mathrm{BZ}$ was sampled by a $5 \times 5 \times 1$ and $7 \times 7 \times 1 \Gamma$-centered $k$-point grid in the Monkhorst-Pack scheme for the structural optimization and the electronic structure calculations, respectively. The effects of van der Waals' interactions were considered through the so-called DFT + D2 method ${ }^{40}$ and we found that DFT + D2 gives essentially the same results as DFT.

\section{Phonon and AIMD}

Structural stability of the material is attested by calculating its phonon band dispersions using the density functional perturbation theory implemented in VASP and the Phonopy ${ }^{41}$, in which a $3 \times 3 \times 1$ supercell was used sampled with a $2 \times 2 \times 1 \quad k$-mesh. The spin-polarized AIMD simulations in the canonical ensemble (NVT) with the Nosé-Hoover thermostat were performed to assess its dynamical stability. In the AIMD simulations, a $2 \times 2 \times 1$ supercell was used, and each was performed for a total duration of $12.0 \mathrm{ps}$ with each time-step of $1.5 \mathrm{fs}$ at $300,500,700$, and $800 \mathrm{~K}$.

\section{Minimum energy path calculations}

In the simulation of the catalyzed chemical reaction, $2 \times 2 \times 1$ supercells were used to model the $2 \mathrm{D}$ catalyst for the adsorption of the various molecules and the determination of the minimum energy pathway and the TSs using the climbing image nudged elastic band (Cl-NEB) method ${ }^{42}$ implemented in VASP. Here, the adsorption energy $E_{\text {ads }}$ of species $i$ is defined by $E_{\text {ads }}(i)=E_{i @ \text { Catalyst }}-E_{\text {Catalyst }}-E_{i}$ where $E_{i @ \text { Catalyst }}$ and $E_{\text {Catalyst }}$ are the total electronic energy of the $2 \mathrm{D}$ catalyst with and without the adsorbate species $i$, and $E_{i}$ is the electronic energy of the species $i$ in the gaseous state.

\section{DATA AVAILABILITY}

The data presented in current study are available from the corresponding author on reasonable request.

Received: 20 July 2020; Accepted: 15 April 2021; Published online: 14 May 2021

\section{REFERENCES}

1. Novoselov, K. S. et al. Electric field effect in atomically thin carbon films. Science 306, 666-669 (2004).

2. Geim, A. K. \& Novoselov, K. S. The rise of graphene. Nat. Mater. 6, 183-191 (2007).

3. Bhimanapati, G. R. et al. Recent advances in two-dimensional materials beyond graphene. ACS Nano 9, 11509-11539 (2015).

4. Gupta, A., Sakthivel, T. \& Seal, S. Recent development in $2 \mathrm{~d}$ materials beyond graphene. Prog. Mater. Sci. 73, 44-126 (2015).

5. Pacilé, D., Meyer, J. C., Girit, Ç. Ö. \& Zettl, A. The two-dimensional phase of boron nitride: few-atomic-layer sheets and suspended membranes. Appl. Phys. Lett. 92, 133107-133109 (2008).
6. Xu, M., Liang, T., Shi, M. \& Chen, H. Graphene-like two-dimensional materials. Chem. Rev. 113, 3766-3798 (2008).

7. Vilé, G. et al. A stable single-site palladium catalyst for hydrogenations. Angew. Chem. Int. Ed. Engl. 54, 11265-11269 (2015).

8. Li, S.-L. et al. Potential of transition metal atoms embedded in buckled monolayer g- $\mathrm{C}_{3} \mathrm{~N}_{4}$ as single-atom catalysts. Phys. Chem. Chem. Phys. 19, 30069-30077 (2017).

9. Choi, W. et al. Recent development of two-dimensional transition metal dichalcogenides and their applications. Mater. Today 20, 116-130 (2017).

10. Manzeli, S., Ovchinnikov, D., Pasquier, D., Yazyev, O. V. \& Kis, A. $2 d$ transition metal dichalcogenides. Nat. Rev. Mater. 2, 17033 (2017).

11. Yang, L. M. et al. Two-dimensional $\mathrm{Cu}_{2} \mathrm{Si}$ monolayer with planar hexacoordinate copper and silicon bonding. J. Am. Chem. Soc. 137, 2757-2762 (2015).

12. Yam, K. M., Guo, N. \& Zhang, C. Two-dimensional $\mathrm{Cu}_{2} \mathrm{Si}$ sheet: a promising electrode material for nanoscale electronics. Nanotechnology 29, 245704-245707 (2018).

13. Zhou, J. \& Sun, Q. Magnetism of phthalocyanine-based organometallic single porous sheet. J. Am. Chem. Soc. 133, 15113-15119 (2011).

14. Tan, J., Li, W., He, X. \& Zhao, M. Stable ferromagnetism and half-metallicity in twodimensional polyporphyrin frameworks. RSC Adv. 3, 7016-7022 (2013).

15. Rodríguez-Manzo, J. A., Cretu, O. \& Banhart, F. Trapping of metal atoms in vacancies of carbon nanotubes and graphene. ACS Nano 4, 3422-3428 (2010).

16. Sun, Q., Wang, Q., Jena, P. \& Kawazoe, Y. Clustering of Ti on a $C_{60}$ surface and its effect on hydrogen storage. J. Am. Chem. Soc. 127, 14582-14583 (2005).

17. Gan, Y., Sun, L. \& Banhart, F. One- and two-dimensional diffusion of metal atoms in graphene. Small 4, 587-591 (2008).

18. Markevich, A. V., Baldoni, M., Warner, J. H., Kirkland, A. I. \& Besley, E. Dynamic behavior of single fe atoms embedded in graphene. J. Phys. Chem. C. 120, 21998-22003 (2016).

19. Yam, K. M., Guo, N., Jiang, Z., Li, S. \& Zhang, C. Graphene-based heterogeneous catalysis: role of graphene. Catalysts 10, 53 (2020).

20. Zhang, L. et al. Graphene defects trap atomic ni species for hydrogen and oxygen evolution reactions. Chem 4, 285-297 (2018).

21. Yan, H. et al. Atomic engineering of high-density isolated co atoms on graphene with proximal-atom controlled reaction selectivity. Nat. Commun. 9, 3197 (2018).

22. Gibertini, M., Koperski, M., Morpurgo, A. F. \& Novoselov, K. S. Magnetic 2d materials and heterostructures. Nat. Nanotechnol. 14, 408-419 (2019).

23. Huang, B. et al. Layer-dependent ferromagnetism in a van der waals crystal down to the monolayer limit. Nature 546, 270-273 (2017).

24. Bonilla, M. et al. Strong room-temperature ferromagnetism in $\mathrm{VSe}_{2}$ monolayers on van der waals substrates. Nat. Nanotechnol. 13, 289-293 (2018).

25. Krasheninnikov, A. V., Lehtinen, P. O., Foster, A. S., Pyykko, P. \& Nieminen, R. M. Embedding transition-metal atoms in graphene: structure, bonding, and magnetism. Phys. Rev. Lett. 102, 126807 (2009).

26. Rivera-Cárcamo, C. \& Serp, P. Single atom catalysts on carbon-based materials. ChemCatChem 10, 5058-5091 (2018).

27. Li, Q., Yang, C., Wu, L., Wang, H. \& Cui, X. Converting benzene into Y-graphyne and its enhanced electrochemical oxygen evolution performance. J. Mater. Chem. A 7, 5981-5990 (2019).

28. Gao, X., Liu, H.-B., Wang, D. \& Zhang, J. Graphdiyne: synthesis, properties, and applications. Chem. Soc. Rev. 48, 908-936 (2019).

29. Li, Q. et al. Synthesis of $\gamma$-graphyne by mechanochemistry and its electronic structure. Carbon 136, 248-254 (2018).

30. He, J. et al. Magnetic properties of single transition-metal atom absorbed graphdiyne and graphyne sheet from DFT+U calculations. J. Phys. Chem. C. 116, 26313-26321 (2012).

31. Birch, F. Finite elastic strain of cubic crystals. Phys. Rev. 71, 809-824 (1947).

32. Kim, C.-W., Kang, S.-H. \& Kwon, Y.-K. Rigid unit modes in $s p-s p^{2}$ hybridized carbon systems: origin of negative thermal expansion. Phys. Rev. B 92, 245434-245439 (2015).

33. Xie, W. H., Xu, Y. Q., Liu, B. G. \& Pettifor, D. G. Half-metallic ferromagnetism and structural stability of zincblende phases of the transition-metal chalcogenides. Phys. Rev. Lett. 91, 037204 (2003).

34. Ding, Y. \& Ni, J. Electronic structures of silicon nanoribbons. Appl. Phys. Lett. 95, 083115 (2009).

35. Sugahara, S. \& Tanaka, M. A spin metal-oxide-semiconductor field-effect transistor using half-metallic ferromagnet contacts for the source and drain. Appl. Phys. Lett. 84, 2307 (2004).

36. Kresse, G. \& Hafner, J. Ab initio molecular dynamics for liquid metals. Phys. Rev. $B$ 47, 558-561 (1993).

37. Kresse, G. \& Furthmüller, J. Efficient iterative schemes for Ab initio total-energy calculations using a plane-wave basis set. Phys. Rev. B 54, 11169-11186 (1996).

38. Blöchl, P. E. Projector augmented-wave method. Phys. Rev. B 50, 17953-17979 (1994).

39. Perdew, J. P., Burke, K. \& Ernzerhof, M. Generalized gradient approximation made simple. Phys. Rev. Lett. 77, 3865-3868 (1996). 
40. Grimme, S. Semiempirical GGA-type density functional constructed with a long range dispersion correction. J. Comp. Chem. 27, 1787-1799 (2006).

41. Togo, A. \& Tanaka, I. First principles phonon calculations in materials science. Scr. Mater. 108, 1-5 (2015).

42. Henkelman, G., Uberuaga, B. P. \& Jónsson, H. A climbing image nudged elastic band method for finding saddle points and minimum energy paths. J. Chem. Phys. 113, 9901-9904 (2000).

\section{ACKNOWLEDGEMENTS}

We acknowledge the support from Ministry of Education of Singapore (R-723-000029-112), NUS academic research fund (R-144-000-410-114) and NUS green energy program (R-143-000-A63-114). Computational works were performed at the NUS Graphene Research Centre computing cluster facilities. The support provided by China Scholarship Council (no. 201807000028) is acknowledged by SL.

\section{AUTHOR CONTRIBUTIONS}

C.Z. supervised the project. S.L. and K.Y. performed all calculations. K.Y. and C.Z. wrote the paper. All authors contributed to data analysis and have given approval to the final version of the manuscript.

\section{COMPETING INTERESTS}

The authors declare no competing interests.

\section{ADDITIONAL INFORMATION}

Supplementary information The online version contains supplementary material available at https://doi.org/10.1038/s41699-021-00235-y.

Correspondence and requests for materials should be addressed to Y.Z. or C.Z.

Reprints and permission information is available at http://www.nature.com/ reprints

Publisher's note Springer Nature remains neutral with regard to jurisdictional claims in published maps and institutional affiliations.

Attribution 4.0 International License, which permits use, sharing, adaptation, distribution and reproduction in any medium or format, as long as you give appropriate credit to the original author(s) and the source, provide a link to the Creative Commons license, and indicate if changes were made. The images or other third party material in this article are included in the article's Creative Commons license, unless indicated otherwise in a credit line to the material. If material is not included in the article's Creative Commons license and your intended use is not permitted by statutory regulation or exceeds the permitted use, you will need to obtain permission directly from the copyright holder. To view a copy of this license, visit http://creativecommons. org/licenses/by/4.0/.

(c) The Author(s) 2021 